\title{
Shikonin Enhances the Antitumor Effect of Cabazitaxel in Prostate Cancer Stem Cells and Reverses Cabazitaxel Resistance by Inhibiting the Expression of ABCG2 and ALDH3A1
}

\section{Lili Wang}

Ludwig-Maximilians-Universitat Munchen

\section{Birgit Stadlbauer}

Ludwig-Maximilians-Universität München

\section{Chen Lyu}

Ludwig-Maximilians-Universitat Munchen

\section{Alexander Buchner}

Ludwig-Maximilians-Universitat Munchen

Heike Pohla ( $\square$ heike.pohla@med.uni-muenchen.de)

Ludwig-Maximilians-Universitat Munchen https://orcid.org/0000-0001-8296-2059

\section{Research}

Keywords: Prostate cancer, Cancer stem cells, Shikonin, Cabazitaxel, ABCG2, ALDH3A1, drug-resistance

Posted Date: August 17th, 2020

DOI: https://doi.org/10.21203/rs.3.rs-58823/v1

License: (c) (i) This work is licensed under a Creative Commons Attribution 4.0 International License.

Read Full License 


\section{Abstract}

Background: Cancer stem cells (CSCs) are a small population among cancer cells, defined as capable of self-renewal, and driving tumor growth, metastasis, and therapeutic relapse. The development of therapeutic strategies to target CSCs is of great importance to prevent tumor metastasis and relapse. Increasing evidence shows that shikonin has inhibiting effects on CSCs. This study was to determine the effect of shikonin on prostate CSCs, and on drug resistant cells.

Methods: Sphere formation assay was used to enrich prostate CSCs. The effect of shikonin on viability, proliferation, migration, and invasion was studied. Typical CSCs markers were analyzed by flow cytometry and RT-qPCR. The cytotoxic mechanism of shikonin was analyzed by staining for annexin V, reactive oxygen species (ROS) and mitochondrial membrane potential. To study the effect of shikonin on drug resistant cells a cabazitaxel resistant cell line was established.

Results: Shikonin inhibited the viability, proliferation, migration, and invasion of prostate CSCs. Shikonin enhanced the antitumor effect of cabazitaxel, which is a second-line chemotherapeutic drug in advanced prostate cancer. Shikonin induced apoptosis through generating ROS and disrupting the mitochondrial membrane potential. Furthermore, shikonin suppressed the expression of ALDH3A1 and ABCG2 in prostate CSCs, which are two markers related to drug-resistance. When inhibiting the expression of ABCG2 and ALDH3A1, the cabazitaxel resistant cells acquired more sensibility to cabazitaxel.

Conclusions: Shikonin enhances the cytotoxic activity of cabazitaxel in prostate CSCs and reverses the cabazitaxel-resistant state.

\section{Background}

Prostate cancer (PCa) is the second most common malignancy disease after lung cancer among males worldwide. The incidence of prostate cancer ranks first among men in developed countries. There were almost 1.3 million new cases of prostate cancer and 359,000 associated deaths worldwide in 2018, ranking as the fifth leading cause of cancer death in men [1]. The PCa patients are initially treated by prostatectomy. After that, the patients are treated by first-line hormonal therapy using Gonadotropinreleasing hormone $(\mathrm{GnRH})$ analogs to inhibit androgen receptor signaling pathways [2]. Once the castration-resistant prostate cancer (CRPC) condition has occurred, patients will receive chemotherapy, including docetaxel and cabazitaxel, or other androgen-modulating agents such as abiraterone acetate and enzalutamide [3]. However, most patients develop resistance to these agents, and the vast majority of CRPC patients develop metastases. Among nonmetastatic CRPC men, nearly $60 \%$ developed metastatic disease during the five years, with most of the metastasis occurring within the first three years [4]. Evidence has shown that the ability for tumor growth and resistance to therapies lies in a small subpopulation of undifferentiated cells called tumor-initiating cells or cancer stem cells (CSCs). Like other kinds of stem cells, they also have the capacity of self-renewal and can form proliferating progenitor cells and differentiated non-dividable tumor cells. Furthermore, they are responsible for the invasion and 
metastasis of tumors $[5,6]$. CSCs can be detected by specific cell surface markers, like ABCG2, ALDH, CD117, CD133, CD44, or CXCR4. These markers drive the PCa progression, therapeutic resistance, and bone metastasis [7]. ATP-binding cassette G2 (ABCG2) profoundly expresses in PCa stem cells (PCSCs), belongs to $A B C$ transporter family, which applies the energy from hydrolysis of ATP to efflux antitumor chemotherapeutic agents from the cancer cells [8], then maintains the concentration of drug under a toxic level [9]. This type of drug resistance is called multiple drug resistance (MDR). ABCG2 is one of the essential MDR proteins, also called breast cancer resistance protein (BCRP) [10]. ABCG2 causes cancer cells to develop resistance to a variety of chemotherapeutics, including taxanes, mitoxantrone, doxorubicin, and tyrosine kinase inhibitors [11]. CSCs in different cancer types show a high expression of ABCG2 providing a high level of drug resistance [12]. The blockage of ABCG2 using the inhibitor, Ko143, enhances the antitumor activities [13]. Besides this, aldehyde dehydrogenase (ALDH) is also a marker for CSCs, related to drug resistance [14]. The human genome consists of $19 A L D H s$, including six $A L D H 1$ and four $A L D H 3$ families. The ALDH molecules play a critical role in the maintenance of detoxification through oxidizing aldehydes to their corresponding carboxylic acids [15]. ALDH3A1, one of the ALDH molecules is a marker of PCSCs, whose expression correlates with PCa progression. In recent years, there has been an increasing interest in Chinese medicines. Shikonin is one of these Chinese medicines, which is derived from the roots of lithospermum erythrorhizon, and demonstrates antioxidant, antiinflammatory, anti-thrombotic, and anticancer effects [16-18]. Shikonin promotes autophagy through inhibition of AKT signaling pathway $[19,20]$, and inhibits the growth of human prostate cancer cells via modulation of the androgen receptor [21]. Shikonin also acts as a tubulin inhibitor to arrest cancer cells in the G2/M phases [22]. Cabazitaxel is a second-line agent given after docetaxel-resistance in CRPC [23], and is still active in patients treated with more than 10 cycles of docetaxel [24]. Once the CRPC patients are resistant to cabazitaxel, there are limited therapeutic options except for symptomatic and supportive treatment. Thus, it is important to find ways to inhibit PCSCs and reverse the cabazitaxel-resistant state.

In this study the effect of shikonin on PCSCs and on cabazitaxel-resistant PCa cells was analyzed. Results show that shikonin improves the toxic effects of cabazitaxel and reverses the cabazitaxelresistant state. It indicates that combining shikonin and cabazitaxel could be a new treatment option for patients with CRPC.

\section{Material And Methods}

\section{Cell lines and cell culture}

The PCa cell lines DU145, derived from a metastatic brain lesion and PC-3, derived from a metastatic bone marrow lesion, were purchased from the DSMZ German Collection of Microorganisms and Cell Culture $\mathrm{GmbH}$. The cell lines were cultured in RPMI 1640 medium containing 10\% fetal calf serum (FCS "Gold Plus", Bio\&Sell, GmbH, Feucht, Germany), 2 mM L-glutamine, $1 \mathrm{mM}$ sodium pyruvate, and 1\% minimal essential medium (Invitrogen, Life Technologies $\mathrm{GmbH}$, Darmstadt, Germany) at $37{ }^{\circ} \mathrm{C}$ in a $5 \%$ $\mathrm{CO}_{2}$ atmosphere. CSCs were generated in DMEM/F12 culture medium supplemented with $2 \% \mathrm{~B}-27$ 
(Invitrogen), $10 \mathrm{ng} / \mathrm{ml}$ human recombinant basic fibroblast growth factor (bFGF, Sigma Aldrich Chemie $\mathrm{GmbH}$, Taufkirchen, Germany), and $10 \mathrm{ng} / \mathrm{ml}$ epidermal growth factor (EGF, Sigma Aldrich). The cabazitaxel-resistant DU145 cell line was generated by culturing in presence of cabazitaxel starting at a concentration of $1 \mathrm{nM}$. When DU145 was resistant to the cabazitaxel at $1 \mathrm{nM}$, concentration was slowly increased. We cultured DU145 cells with cabazitaxel for at least eight months until a concentration of $6 \mathrm{nM}$ was reached.

\section{Sphere formation assay}

Sphere formation was conducted to generate the PCSCs. DU145 and PC-3 cells were harvested using StemPro ${ }^{\circledR}$ Accutase ${ }^{\circledR}$ (Life Technologies, Thermo Fisher Scientific, Waltham, MA, USA) and seeded in $75 \mathrm{~cm}^{2}$ ultra-low attachment flasks (Corning Costar, Amsterdam, The Netherlands) in $10 \mathrm{ml}$ CSCs medium. After seven days, spheres were harvested into a $15 \mathrm{ml}$ tube, centrifuged at $500 \mathrm{~g}$ for 4 minutes at room temperature, and dissociated using Accutase for 10 minutes at $37^{\circ} \mathrm{C}$. After centrifugation, cells were filtered through a cell strainer with $40 \mu \mathrm{M}$ nylon mesh (BD Biosciences, Heidelberg, Germany), counted, and used for different assays. For enrichment of CSCs, the spheres were dissociated and a second round of sphere formation was done.

\section{Drug sensitivity assay}

Cell viability was assessed using the CellTiter-Blue Cell Viability Assay (Promega, Mannheim, Germany) according to the manufacturer's instructions. Cells were seeded on a 96-well microtiter plate (1500 cells/well) and treated with different concentrations of shikonin or cabazitaxel (both from Selleckchem, Houston, Texas, USA). After $24 \mathrm{~h}$ and $48 \mathrm{~h}$, measurement of fluorescence was done at 560(20) excitation/590(10) emission using the FLUOstar OPTIMA microplate reader (BMG LABTECH, Ortenberg, Germany) and the OPTIMA software version 2.0. The logit regression model was used to calculate the half-maximal inhibitory concentration (IC50).

\section{Cell proliferation assay}

Proliferation was evaluated using the CellTiter $96 \mathrm{AQ}_{\text {ueous }}$ One Solution Cell Proliferation Assay (Promega $\mathrm{GmbH}$, Mannheim, Germany) according to the manufacturer's instructions. Cells were seeded in 96-well microtiter plates at $5 \times 10^{3}$ and treated with the drugs. After $24 \mathrm{~h}$ and $48 \mathrm{~h}$, the reagent was added directly to the wells, the plates incubated for three hours and then the absorbance was read at $490 \mathrm{~nm}$ with the Emax precision microplate reader (MWG Biotech, Ebersberg, Germany).

\section{Apoptosis assay}

A total of $4 \times 10^{5}$ cells were seeded and treated with or without drugs. After five days, cells were harvested and resuspended in $100 \mu$ Annexin V Binding Buffer. Five microliter APC-Annexin V and $5 \mu \mathrm{l}$ 7aminoactinomycin $D$ (both from BD Biosciences) were added. Following incubation for 15 minutes at room temperature $100 \mu \mathrm{l}$ Annexin V Binding Buffer were added again and the cells measured using the FACSCalibur (Becton Dickinson, San Jose, CA, USA). A minimum of $1 \times 10^{4}$ cell events were recorded per sample. BD CellQuest software (version 4.0.2) was used for data acquisition, and data were analysed 
using FlowJo software (version 9.9.5; Tree Star Inc., Ashland, OR, USA). The Annexin V positive cells were considered as apoptotic cells.

\section{Migration assay}

For cell migration the scratch wound healing assay was applied using 24-well culture plates with small 2well silicone inserts per well building a cell free gap of $500 \mu \mathrm{m}$ (ibidi $\mathrm{GmbH}$, Martinsried, Germany). Cells were diluted to a concentration of $4 \cdot 10^{5} \mathrm{cell} / \mathrm{s} / \mathrm{ml}$, seeded in a volume of $70 \mu \mathrm{l}$ into each culture insert, and incubated at $37{ }^{\circ} \mathrm{C}$ until a confluent cell monolayer was achieved. Drugs were added after the inserts were removed with sterile tweezers. Pictures were taken under a microscope at different time points, 0,3 , $6,9,21,24,27$, and $30 \mathrm{~h}$. The proportion of gap covered by cells at each time was determined and the data were analyzed with the web-based Automated Cellular Analysis System (ACAS, MetaVi Labs, Bottrop, Germany) using FastTrack Al image analysis algorithms.

\section{Invasion assay}

Cell invasion was done using the Boyden Chamber system. The transwell inserts in 24-well plates $(8.0 \mu \mathrm{m}$ pores; Falcon, Corning, New York, NY, USA) were coated with growth factor reduced Matrigel Basement Matrix (Corning; $30 \mu \mathrm{g} / 100 \mu \mathrm{l} /$ insert) and incubated for at least 4 hours at room temperature. Afterwards 30,000 cells were seeded in $250 \mu \mathrm{l}$ serum-free DMEM medium with and without drugs onto the Matrigel. In the lower chamber, $750 \mu \mathrm{l}$ DMEM with 10\% FCS was added. After $48 \mathrm{~h}$ incubation, the upper surface of the transwell membrane was gently wiped with a moistened cotton swab to remove cells that had not migrated. Cells, which had moved through the Matrigel to the lower surface of the membrane were fixed with $4 \%$ paraformaldehyde for 5 minutes and then stained with $1 \%$ crystal violet for 1 minute. After drying, cells were counted (three fields per insert; Fiji ImageJ software [25]).

\section{Determination of aldehyde dehydrogenase}

Active aldehyde dehydrogenase (ALDH) was measured using the ALDEFLUOR kit according to the manufacturer's instructions (StemCell Technologies, Grenoble, France). Brightly fluorescent ALDHexpressing cells were detected in the green fluorescence FL1 channel $(520-540 \mathrm{~nm})$ of the FACSCalibur. The control group included N,N-diethylaminobenzaldehyde (DEAB) at $15 \mu \mathrm{M}$, which is an inhibitor of ALDH activity. BD CellQuest software was used for data acquisition, and data were analysed using FlowJo software.

\section{Measurement of ABCG2 using flow cytometry}

Spheres were dissociated using Accutase and seeded in 6-well low attachment plates with or without shikonin. Twenty-four hours later, cells were stained with APC-conjugated ABCG2 monoclonal antibody (clone 5D3/CD338, BD Biosciences). LIVE/DEAD ${ }^{\circ}$ Fixable Blue Dead Cell Stain Kit (Life Technologies) was used to determine the viability and exclude dead cells during analysis. All measurements were accomplished using the LSRII flow cytometer (BD Biosciences). Data were processed using FlowJo software.

RT-qPCR 
RNA was isolated using the RNeasy Mini-Kit (Qiagen, Hilden, Germany) according to the manufacturer's instructions. A total of $1 \mu \mathrm{g}$ RNA was then used for reverse transcription according to the kit instructions (Promega $\mathrm{GmbH}$ ). Reverse transcription polymerase chain reaction was performed with FastStart Essential DNA Green Master kit (Roche, Penzberg, Germany) and Light Cycler ${ }^{\circledR} 96$ (Roche). The reaction was set up with $2 \mu \mathrm{l}$ cDNA template, $1 \mu \mathrm{l}$ forward primer, $1 \mu \mathrm{l}$ reverse primer, $5 \mu \mathrm{l}$ FastStart Essential DNA Green Master reagent mix, and $1 \mu \mathrm{l} \mathrm{PCR}$ grade water. For amplification, a hot start with $95^{\circ} \mathrm{C}$ for 10 minutes, followed by 40 cycles of denaturation at $95^{\circ} \mathrm{C}$ for 10 seconds, annealing at $60^{\circ} \mathrm{C}$ for 10 seconds, and extension at $72^{\circ} \mathrm{C}$ for 10 seconds were used. Then a melting process with $95^{\circ} \mathrm{C}$ for 10 seconds, $65^{\circ} \mathrm{C}$ for 60 seconds and $97^{\circ} \mathrm{C}$ for 1 second was performed. Data were analyzed by the Lightcyclerß 96 software (1.1 version). The relative expression was calculated using the $2^{-\triangle \triangle C t}$ method. The transcription level of GAPDH and $A C T B$ was used as an internal control. Primers were listed in the Additional file 1 Table S1.

\section{Confocal immunofluorescence microscopy}

Fifty thousand cells per well were seeded in 16-well chambered coverslips (Molecular Probes, Thermo Fisher Scientific) and cultured for $24 \mathrm{~h}$. Then cells were fixed with $4 \%$ paraformaldehyde for 10 minutes, permeabilized with $0.1 \%$ Triton X-100 for 5 minutes, and blocked in $3 \%$ BSA for $1 \mathrm{~h}$. Afterwards cells were incubated with the primary rabbit polyclonal ALDH3A1 antibody (Abcam, Cambridge, UK) at $5 \mu \mathrm{g} / \mathrm{ml}$, and mouse monoclonal ABCG2 antibody (clone BXP-21, Abcam) at a 1/50 dilution overnight at $4{ }^{\circ} \mathrm{C}$. After a three time washing step, the cells were incubated with the secondary antibody goat anti-rabbit IgG $\mathrm{H} \& \mathrm{~L}$ (Alexa Fluor ${ }^{\circledR} 647$ ) preadsorbed (Abcam), and goat anti-mouse IgG H\&L (Alexa Fluor® 488, Abcam) used in a dilution of 1:200 at room temperature for $1 \mathrm{~h}$ in the dark. Cells were then washed three times with PBS and incubated with Molecular Probes ${ }^{\circledR}$ NucBlue ${ }^{\circledR}$ Fixed Cell Stain ReadyProbes ${ }^{\circledR}$ reagent (DAPI special formulation, Thermo Fisher Scientific) for 20 minutes at room temperature in the dark. Ibidi mounting medium (ibidi $\mathrm{GmbH}$ ) was dropped to cover the cell layer, followed by a coverslip. The fluorescence intensity was measured by confocal Leica SP5 for DAPI at $365 \mathrm{~nm}$ excitation and $420 \mathrm{~nm}$ emission wavelengths. The negative control was a secondary-only assay to demonstrate low non-specific binding of the secondary antibody.

\section{Measurement of reactive oxygen species (ROS)}

Cells were seeded in a 96-well microplate (25,000/well) and cultured overnight. Then, $100 \mu \mathrm{l} 25 \mu \mathrm{M} 2^{\prime}, 7^{\prime}-$ dichlorofluorescein diacetate (DCFDA, Abcam) was added for 45 minutes at $37{ }^{\circ} \mathrm{C}$ in the dark. After washing three times, cells were treated with shikonin for $6 \mathrm{~h}$. ROS was measured on the FLUOstar OPTIMA microplate reader at $485 \mathrm{~nm}$ excitation/535 nm emission. ROS generation was rescued by the antioxidant $\mathrm{N}$-acetyl-L-cysteine (NAC) with pretreatment for 4 hours at $1 \mathrm{mM}$.

\section{Measurement of mitochondrial membrane potential}

The integrity of mitochondrial membrane potential $(\Delta \psi \mathrm{m})$ was evaluated using the JC-1 mitochondrial membrane potential kit (Abcam). Briefly, cells were seeded and treated with shikonin for $48 \mathrm{~h}$. Then, cells were stained with $20 \mu \mathrm{M} \mathrm{JC}-1$ (tetraethylbenzimidazolylcarbocyanine iodide) for 10 minutes at $37^{\circ} \mathrm{C}$, 
washed once and measured on the FLUOstar OPTIMA microplate reader. With normal mitochondrial function (high $\Delta \psi \mathrm{m}$ ) the dye aggregates yielding a red to orange emission (590 $\pm 17.5 \mathrm{~nm}$ ), injured mitochondria are depolarized (low $\Delta \psi \mathrm{m}$ ) and leads to monomer formation yielding green emission (530 $\pm 15 \mathrm{~nm}$ ). Signals were recorded in a monomer form at Excitation/Emission equaling to $485 \pm 10 / 590 \mathrm{~nm}$, and in an aggregate form at Excitation/Emission equaling to $485 \pm 10 / 520 \mathrm{~nm}$. A ratio between the monomer and aggregate was obtained and plotted.

\section{Downregulation of ABCG2 and ALDH3A1}

Two inhibitors of $A B C G 2$ and $A L D H 3 A 1$ were used namely Ko143 (Tocris Biosciences, Bio-Techne GmbH, Wiesbaden-Neuenstadt, Germany) at $1 \mu \mathrm{M}$, and CB29 (Sigma Aldrich) at $32 \mu \mathrm{M}$, respectively. Small interfering RNAs (siRNAs) were also used to silence $A B C G 2$ and $A L D H 3 A 1$, which were designed by Ambion (Life Technologies, Thermo Fisher Scientific). The Silencer ${ }^{\circledR}$ Select siRNAs sequences are shown in the Additional file 2 Table S2. Silencer ${ }^{\circledR}$ Select negative control and Silencer ${ }^{\circledR}$ Select GAPDH positive control were also used. For transfection the Lipofectamine RNAiMAX Reagent (Invitrogen, Life Technologies, Thermo Fisher Scientific) was applied as described in the manufacturer's instruction.

\section{Statistical methods}

All experiments were independently performed at least three times. The analyses were carried out using SPSS version 25.0 (IBM, Armonk, NY). All numerical data were expressed in the form of the average of the values (mean), plus the standard error of the mean (SEM). Data from two different groups were compared using the Mann-Whitney $U$ test. The Pearson product-moment correlation coefficient was calculated to determine the correlation between ABCG2 and ALDH3A1 in the confocal immunofluorescence microscopy. All p-values were 2-sided, and $p<0.05$ was considered significant.

\section{Results}

\section{Shikonin inhibits viability and proliferation of PCSCs}

In the first part of the study, we generated PCSCs using sphere formation assay over 2-3 generations. The PCSCs in the ultra-low attachment flasks with serum-free medium formed non-adherent free-floating spheres, while differentiated adherent prostate cancer cells shrank and died [26]. Figure 1a shows the generation of the spheres within 10 days. DU145 and PC-3 and the corresponding PCSCs were treated with different concentrations of shikonin $(0,1.5,3,6,12,24$, and $48 \mu \mathrm{M})$ for $24 \mathrm{~h}$ and $48 \mathrm{~h}$, see Additional file 3 Fig. S1. The results of the drug sensitivity assay show that shikonin inhibited the cell viability in a dose-dependent manner (Fig. 1b). The half-maximal inhibitory concentration (IC50) analyzed by logit regression model using the SPSS software mounted around $0.75 \mu \mathrm{M}$ for DU145, $4 \mu \mathrm{M}$ for DU145 CSCs, 5 $\mu \mathrm{M}$ for PC-3, and $7 \mu \mathrm{M}$ for PC-3 CSCs. Using different concentrations ( $0.5 x$ IC50, IC50, and $2 x$ IC50) an inhibiting effect of shikonin on the proliferation rate was also observed (Fig. $1 \mathrm{c}$ and Additional file 3 Fig. S1). Results showed that DU145 CSCs and PC-3 CSCs were much less sensitive to shikonin than the adherent DU145 and PC-3 cells. It supported the point that cancer stem cells were a special cell population like drug-resistant cells. 


\section{Shikonin inhibits migration and invasion of PCa cells}

Since the migration and invasion ability are two important biological characteristics, we evaluated the effect of shikonin on migration and invasion of DU145, PC-3 and their corresponding sphere cells. Migration was evaluated using the scratch wound healing assay. After treatment with different concentrations of shikonin pictures were taken at different time points. Shikonin inhibited the migration of both the adherent cells and sphere cells to the center of the gap (Fig. 2a-b and Additional file 4 Fig. S2). Invasion ability was determined using the Boyden chamber system. Cells were treated again with different concentrations of shikonin $(0.5 \times$ IC50, $1 \times$ IC50, and $2 \times$ IC50) for 48 hours. The numbers of invaded cells were decreased significantly in the adherent PCa cell lines and even in the corresponding PCSCs, (Fig. 2c-d). It can be concluded that shikonin was able to inhibit migration and invasion remarkably in PCa cells.

\section{Shikonin enhances the antitumor effect of cabazitaxel}

To investigate if shikonin enhances the antitumor effects of cabazitaxel, we performed the viability, proliferation, and invasion assay. First the IC50 of cabazitaxel was determined. DU145 cells were treated with different concentrations of cabazitaxel $(0,1,2,4$, and $8 \mathrm{nM})$ for $48 \mathrm{~h}$. Results showed that cabazitaxel inhibited the viability of DU145 cells in a dose-dependent manner, the IC50 concentration was around $3 \mathrm{nM}$ (see Additional file 5 Fig. S3). Then, DU145 and DU145-CSCs were treated with $0.75 \mu \mathrm{M}$ shikonin combined with different concentrationsd of cabazitaxel for $48 \mathrm{~h}$. Shikonin alone repeatedly resulted in a significant decrease in both viability (Fig. 3a and Additional file 6 Fig. S4) and proliferation (Fig. 3b and Additional file 6 Fig. S4) in DU145 and DU145 CSCs compared to cabazitaxel alone. The effect was more pronounced in the combination treatment, even at a lower concentration of cabazitaxel (<IC50). Similarly, the number of apoptotic cells was higher following treatment with cabazitaxel plus shikonin (Fig. 3c). Also in the invasion assay a significant higher inhibition was observed (Fig. 3d). Altogether, shikonin sensitizes the antitumor effect of cabazitaxel in both DU145 and DU145-CSCs.

\section{Shikonin induces apoptosis through ROS-mitochondria membrane potential apoptosis pathways in PCa cell lines and PCSCs}

The effect of shikonin on the apoptosis rate was also tested in the cell line PC-3 and the corresponding PC-3 CSCs. Results showed that shikonin dramatically induced apoptotic death both in the PCa cell lines DU145 and PC-3 and the corresponding PCSCs (see Additional file 7 Fig. S5).

Reactive oxygen species (ROS) generation is an essential effect of shikonin during cell death in several types of cancers [27-30]. To study the role of shikonin in prostate cancer cells we used the cell permeable reagent $2,7^{\prime}$ - dichlorofluorescein diacetate (DCFDA), a fluorogenic dye that measures ROS activity within the cell. After diffusion into the cell, DCFDA is deacetylated by cellular esterases to a nonfluorescent compound, which is later then oxidized by ROS into 2', 7' - dichlorofluorescein (DCF), a highly fluorescent substance. Treatment with shikonin for 6 hours dramatically enhanced ROS generation in the PCa cell lines and the corresponding PCSCs (Fig. 4a). When the cells were pretreated with the ROS 
scavenger, $\mathrm{N}$-acetyl-L-cysteine (NAC), at $1 \mathrm{mM}$ for $4 \mathrm{~h}$ the effect of shikonin was significantly reduced as shown in the viability assay, which proves that shikonin induces apoptosis via ROS generation (Fig. 4b).

Then we conducted staining with tetraethylbenzimidazolylcarbocyanine iodide (JC-1) to investigate if shikonin disrupted the mitochondrial membrane potential of PCa cells and PCSCs. According to the kit instructions due to low mitochondrial membrane potential, JC-1 is predominantly a monomer that yields green fluorescence with emission of $530 \pm 15 \mathrm{~nm}$. At high concentrations (due to high mitochondrial membrane potential), the JC-1 aggregates

yielding a red to orange colored fluorescence $(590 \pm 17.5 \mathrm{~nm})$ Both monomer and aggregate forms were measured, and a ratio between the two measurements was obtained and plotted. Exposure to shikonin for $48 \mathrm{~h}$ dramatically reduced aggregates fluorescence, indicating that shikonin induced apoptosis through ROS formation and triggered the disruption of the mitochondrial membrane potential. NAC reversed mitochondrial dysfunction and verified our conclusion (Fig. 4c). Besides this, cabazitaxel alone cannot influence ROS generation in DU145 cells (Fig. 4d). It suggested that cabazitaxel cannot interrupt the antitumor effect of shikonin.

\section{Shikonin inhibits the expression of ABCG2 and ALDH3A1 in PCSCs.}

There are different cancer stem cell markers known for prostate cancer driving drug-resistance, and cancer relapse as for example ABCG2, ALDH, CD44, CXCR4 [7]. Figure 5a and Additional file 8 Fig. S6 demonstrates that shikonin inhibited the expression of both ALDH and ABCG2 (CD338) shown by flow cytometry. Similarly, RT-qPCR showed that shikonin inhibited the expression of $A L D H 3 A 1$ and $A B C G 2$ (Fig. 5b). In some publications it was reported that increased expression of the drug transporter gene $A B C G 2$, was detected in the ALDH + cell population [31,32]. Inhibition of ALDH1A1 activity decreased the expression of $A B C G 2$ and reduced chemotherapy resistance in ovarian cancer cell lines [33].To explore if shikonin downregulates the expression of $A B C G 2$ through blocking the expression of $A L D H 3 A 1$, we silenced $A L D H 3 A 1$ using siRNA for $48 \mathrm{~h}$ and tested $A B C G 2$ expression by RT-qPCR. The results showed that downregulation of $A L D H 3 A 1$ could not influence the expression of $A B C G 2$ (Fig. 5d). However, in the confocal fluorescence microscopy it was observed that ALDH3A1 was overlapped with ABCG2 in DU145 CSCs (Fig. 5c). Both the Pearson correlation coefficient and the overlap coefficient were 0.9 analyzed by the colocalization finder of image $\mathrm{J}$ software (Fig. $5 \mathrm{~d}$ ). It can be suggested that ALDH3A1 and ABCG2 were at least co-expressed in DU145 CSCs. To investigate the relation between ABCG2 and ALDH3A1, we silenced the $A B C G 2$. After downregulating $A B C G 2$, the expression of $A L D H 3 A 1$ decreased (Fig. $5 \mathrm{e}$ ). This implies that shikonin can block the expression of ALDH3A1 by inhibiting ABCG2.

\section{Shikonin reverses the resistant-state of caba-DU145 through inhibiting the expression of ABCG2 and ALDH3A1}

In clinical practice, cabazitaxel has proved to be effective for docetaxel-resistant CRPC patients [24]. When the CRPC patients become resistant to cabazitaxel, there are few therapeutic options left. According to our results, ABCG2 and ALDH3A1 are two primary cancer stem cell markers that can drive 
drug-resistance and their expression can be regulated by shikonin. Then we wanted to know if shikonin can abolish the cabazitaxel-resistant state by inhibiting the expression of ABCG2 and ALDH3A1. We generated a cabazitaxel-resistant DU145 cell line (caba-DU145) by gradually increasing the dose of cabazitaxel over eight months (see Additional file 9 Fig. S7). Then the caba-DU145 cells were pretreated with the ABCG2 inhibitor Ko143 at $1 \mu \mathrm{M}$. After five days, the cell viability of caba-DU145 response to cabazitaxel was determined. Results showed that after treatment with Ko143 the cells were more sensitive to cabazitaxel compared to the untreated cells (Fig. 6a). The same was observed using the proliferation assay (Fig. 6b). Also, treatment with the ALDH3A1 inhibitor CB29, showed a higher sensitivity of the cells to cabazitaxel compared to the untreated cells (Fig. 6c). The same effect was observed again using the proliferation assay (Fig. 6d). Furthermore, inhibiting ALDH3A1 and ABCG2 using CB29 and Ko143, decreased the apoptosis rate of caba-DU145 cells (Fig. 6e). As we expected, using low concentration of shikonin at $0.375 \mu \mathrm{M}$ in combination with cabazitaxel at $3 \mathrm{nM}$ increased the apoptotic rate compared to cabazitaxel alone in the caba-DU145 cells (Fig. 6f).

Our results suggested that shikonin not only enhances the antitumor effect of cabazitaxel but also reverses the cabazitaxel-resistant state by inhibiting the expression of ABCG2 and ALDH3A1, and ABCG2 suppresses the expression of ALDH3A1.

\section{Discussion}

Prostate cancer is a highly malignant carcinoma, due to its late detection and therapeutic-resistant caused relapse. Although androgen deprivation treatment is effective at the beginning of advanced PCa [34], half of those cases become resistant to androgen deprivation treatment [35]. The taxane docetaxel is the first-line agent for CRPC, but most patients acquire resistance and disease relapse. Then cabazitaxel, a second-line taxane, is used for patients who developed resistance to docetaxel [23]. It is urgent to find some new agents for the treatment of CRPC, and to reveal the mechanisms of cabazitaxel resistance. Recently, it was proven that shikonin shows antitumor effects in human glioblastoma stem cells [36], and prostate cancer $[19,21,37]$. In our study shikonin targeted not only normal prostate cancer cell lines but also PCSCs, which are responsible for drug resistance [5]. Several mechanisms have been discussed for drug resistance in CSCs. First, the CSCs stay in the resting phase of the cell cycle, losing sensitivity to chemotherapeutic drugs because the majority of agents mainly target cells in the proliferating phase. Second, CSCs express efflux transporters mostly belonging to the ATP-binding cassette gene family (ABC transporters). These transporters pump chemotherapeutic drugs out of the CSCs [8]. Also, the hypoxic CSCs microenvironment is one leading cause of drug resistance. This microenvironment protects CSCs to hide in a quiescent state in the tissue and avoid the attack of drugs $[38,39]$. Since shikonin attacks the CSCs, we guessed that it also inhibits the cabazitaxel resistance. We tested different cancer stem cell markers in RT-qPCR, including CD44, TROP2, ALDH1A1, ALDH2, ALDH3A1, and ABCG2 (Fig. 5b and Additional file $10 \mathrm{Fig}$. S8), and found that shikonin downregulated the expression of $A B C G 2$ and $A L D H 3 A 1$ in PCSCs. In addition, blocking of $A B C G 2$ reduced $A L D H 3 A 1$ expression and can regulate those pathways reversing the resistant state. 
In our studies shikonin attacks PCSCs by inhibiting proliferation, migration, invasion, and by promoting apoptosis. It was further demonstrated that apoptosis induced by shikonin in prostate cancer stem cells was activated through the ROS-mitochondria membrane potential pathway as earlier discussed for normal PCa cell lines [40].

$A B C G 2$ is one of the $A B C$ transporters, which drives CSCs resistance through efflux drugs. The ABCG2+ cancer cells initially called side population cells based on flow cytometry results, which can efflux most agents and prevent treatment success [12]. Inhibition of ABCG2 increased the chemotherapeutic agents' sensitivity in breast cancer cells [41], and ovarian carcinoma cells [42].

Cancer cells with high ALDH expression display elevated tumorigenicity capacity, and have a critical role in regulating self-renewal and differentiation. Therefore, high ALDH activity is known as a characteristic feature of CSCs [43]. ALDH3A1, which is one of the ALDH isoforms, is highly expressed in PCSCs and its expression is related to PCa progression [44]. A dramatic elevation of ALDH3A1 is observed in DU145 cellderived lung metastasis, metastatic lymph node, and bone metastasis compared to local xenograft tumors. ALDH3A1 highly expressed in DU145 spheres contrasted with adherent DU145 cells [44]. Inhibition of ALDH3A1 increased the drug-sensitivity of lung adenocarcinoma, glioblastoma cell lines [45], and head and neck squamous cell carcinoma [46].

Shikonin downregulated both ABCG2 and ALDH3A1 in PCSCs, which were likely to correlate to cabazitaxel resistance, and ABCG2 can inhibit the expression of ALDH3A1. When blocking ABCG2 and ALDH3A1, the caba-DU145 cells are anew sensitive to cabazitaxel. Altogether, we concluded that shikonin can reverse the cabazitaxel-resistant state by blocking the expression of ABCG2 and ALDH3A1, and ALDH3A1 themselves can be inhibited by suppressing ABCG2.

From the literature it can be concluded, that the antitumor attack of shikonin will be exerted via blocking the PI3K/AKT signaling pathway $[47,48]$. Also, this pathway regulates the expression of ABCG2 and confers resistance to chemotherapy. The blockage of the PI3K/AKT pathway enhances the sensitivity to chemotherapeutic agents $[49,50]$. Therefore, we supposed that shikonin reversed the drug-resistant state through modulating the PI3K/AKT-ABCG2-ALDH3A1 axis in PCSCs.

Therefore, shikonin can sensitize both PCa cells and PCSCs may lead to a better therapeutic option in the future. However, a further preclinical in vivo study with shikonin and cabazitaxel is necessary to verify these in vitro findings. Also, the results indicate that some potential inhibitors of ABCG2 and ALDH3A1 can be applied to patients with chemotherapy resistance, and further researches are needed.

\section{Conclusions}

In summary, our results reveal for the first time that shikonin attacks PCSCs through ROS-mitochondria membrane potential apoptosis pathways, and cabazitaxel cannot influence this process. Shikonin reverses the cabazitaxel-resistant state by inhibiting the expression of ABCG2 and ALDH3A1 or by inhibiting ALDH3A1 through suppressing ABCG2 (Fig. 6g). The combination of shikonin and cabazitaxel 
has a synergistic effect. It enables the use of a lower dose of cabazitaxel and might be a new therapeutic option for CRPC with perhaps lower side effects.

\section{Abbreviations}

CSCs cancer stem cells; PCSCs:prostate cancer stem cells; PCa:prostate cancer; CRPC:castration-resistant prostate cancer; caba-DU145:cabazitaxel-resistant DU145; ROS:reactive oxygen species; ABCG2:ATPbinding cassette G2; ALDH3A1:Aldehyde dehydrogenase 3A1; ALDH:aldehyde dehydrogenase;

\section{Declarations}

Acknowledgments

Not applicable.

Funding

The study was supported by the LMU clinic. The author Lili Wang is funded by the China scholarship council.

Availability of data and materials

All data generated or analysed during this study are included in this published article and its supplementary information files.

Authors' contributions

LW performed the experiments, analysed the data and was responsible for writing the manuscript, BS provided technical support, $C L$ discussed the experimental approaches. $A B$ was involved in analyzing the data. HP was responsible for the conception and design of the study, analyzing the data and writing the manuscript. All authors read and approved the final manuscript.

Competing interests

The authors declare that they have no competing interests.

Consent for publication

Not applicable

Ethics approval and consent to participate

Not applicable 
Publisher's Note

Author Details

${ }^{1}$ Tumor Immunology Laboratory, LIFE Center, LMU Klinikum, University Munich, Germany.

${ }^{2}$ Department of Urology, LMU Klinikum, University Munich, Germany.

\section{References}

1. Bray F, Ferlay J, Soerjomataram I, Siegel RL, Torre LA, Jemal A. Global cancer statistics 2018: GLOBOCAN estimates of incidence and mortality worldwide for 36 cancers in 185 countries. CA Cancer J Clin. 2018;68:394-424.

2. Kahn B, Collazo J, Kyprianou N. Androgen receptor as a driver of therapeutic resistance in advanced prostate cancer. Int J Biol Sci. 2014;10:588-95.

3. Tucci M, Caffo O, Buttigliero C, Cavaliere C, D'Aniello C, Di Maio M, Kinspergher S, Maines F, Rizzo M, Rossetti S, et al. Therapeutic options for first-line metastatic castration-resistant prostate cancer: Suggestions for clinical practise in the CHAARTED and LATITUDE era. Cancer Treat Rev. 2019;74:3542.

4. Moreira DM, Howard LE, Sourbeer KN, Amarasekara HS, Chow LC, Cockrell DC, Hanyok BT, Aronson WJ, Kane CJ, Terris MK, et al. Predictors of Time to Metastasis in Castration-resistant Prostate Cancer. Urology. 2016;96:171-6.

5. Kreso A, Dick JE. Evolution of the cancer stem cell model. Cell Stem Cell. 2014;14:275-91.

6. Lobo NA, Shimono Y, Qian D, Clarke MF. The biology of cancer stem cells. Annu Rev Cell Dev Biol. 2007;23:675-99.

7. Harris KS, Kerr BA. Prostate Cancer Stem Cell Markers Drive Progression, Therapeutic Resistance, and Bone Metastasis. Stem Cells Int 2017, 2017:8629234.

8. Stavrovskaya AA. Cellular mechanisms of multidrug resistance of tumor cells. Biochemistry. 2000;65:95-106.

9. Leonard GD, Fojo T, Bates SE. The role of ABC transporters in clinical practice. Oncologist. 2003;8:411-24.

10. Cojoc M, Mabert K, Muders MH, Dubrovska A. A role for cancer stem cells in therapy resistance: cellular and molecular mechanisms. Semin Cancer Biol. 2015;31:16-27.

11. An Y, Ongkeko WM. ABCG2: the key to chemoresistance in cancer stem cells? Expert Opin Drug Metab Toxicol. 2009;5:1529-42.

12. Patrawala L, Calhoun T, Schneider-Broussard R, Zhou J, Claypool K, Tang DG. Side population is enriched in tumorigenic, stem-like cancer cells, whereas ABCG2 + and ABCG2- cancer cells are similarly tumorigenic. Cancer Res. 2005;65:6207-19. 
13. Stacy AE, Jansson PJ, Richardson DR. Molecular pharmacology of ABCG2 and its role in chemoresistance. Mol Pharmacol. 2013;84:655-69.

14. Moreb JS. Aldehyde dehydrogenase as a marker for stem cells. Curr Stem Cell Res Ther. 2008;3:23746.

15. Vasiliou V, Pappa A, Petersen DR. Role of aldehyde dehydrogenases in endogenous and xenobiotic metabolism. Chem Biol Interact. 2000;129:1-19.

16. Chen X, Yang L, Oppenheim JJ, Howard MZ. Cellular pharmacology studies of shikonin derivatives. Phytother Res. 2002;16:199-209.

17. Wei PL, Tu CC, Chen CH, Ho YS, Wu CT, Su HY, Chen WY, Liu JJ, Chang YJ. Shikonin suppresses the migratory ability of hepatocellular carcinoma cells. J Agric Food Chem. 2013;61:8191-7.

18. Wang Y, Zhou Y, Jia G, Han B, Liu J, Teng Y, Lv J, Song Z, Li Y, Ji L, et al. Shikonin suppresses tumor growth and synergizes with gemcitabine in a pancreatic cancer xenograft model: Involvement of NFkappaB signaling pathway. Biochem Pharmacol. 2014;88:322-33.

19. Chen Y, Zheng L, Liu J, Zhou Z, Cao X, Lv X, Chen F. Shikonin inhibits prostate cancer cells metastasis by reducing matrix metalloproteinase-2/-9 expression via AKT/mTOR and ROS/ERK1/2 pathways. Int Immunopharmacol. 2014;21:447-55.

20. Lee MJ, Kao SH, Hunag JE, Sheu GT, Yeh CW, Hseu YC, Wang CJ, Hsu LS. Shikonin time-dependently induced necrosis or apoptosis in gastric cancer cells via generation of reactive oxygen species. Chem Biol Interact. 2014;211:44-53.

21. Jang SY, Jang EH, Jeong SY, Kim JH. Shikonin inhibits the growth of human prostate cancer cells via modulation of the androgen receptor. Int J Oncol. 2014;44:1455-60.

22. Guo J, Chen XF, Liu J, Lin HY, Han HW, Liu HC, Huang SC, Shahla BK, Kulek A, Qi JL, et al. Novel shikonin derivatives targeting tubulin as anticancer agents. Chem Biol Drug Des. 2014;84:603-15.

23. Al-Mansouri L, Gurney H. Clinical concepts for cabazitaxel in the management of metastatic castration-resistant prostate cancer. Asia Pac J Clin Oncol 2019.

24. Shiota M, Nakamura M, Yokomizo A, Tomoda T, Sakamoto N, Seki N, Hasegawa S, Yunoki T, Harano M, Kuroiwa K, Eto M. Efficacy and safety of cabazitaxel for castration-resistant prostate cancer in patients with > 10 cycles of docetaxel chemotherapy: a multi-institutional study. Med Oncol. 2019;36:32.

25. Schindelin J, Arganda-Carreras I, Frise E, Kaynig V, Longair M, Pietzsch T, Preibisch S, Rueden C, Saalfeld S, Schmid B, et al. Fiji: an open-source platform for biological-image analysis. Nat Methods. 2012;9:676-82.

26. Abbaszadegan MR, Bagheri V, Razavi MS, Momtazi AA, Sahebkar A, Gholamin M. Isolation, identification, and characterization of cancer stem cells: A review. J Cell Physiol. 2017;232:2008-18.

27. Chang IC, Huang YJ, Chiang TI, Yeh CW, Hsu LS. Shikonin induces apoptosis through reactive oxygen species/extracellular signal-regulated kinase pathway in osteosarcoma cells. Biol Pharm Bull. 2010;33:816-24. 
28. Singh F, Gao D, Lebwohl MG, Wei H. Shikonin modulates cell proliferation by inhibiting epidermal growth factor receptor signaling in human epidermoid carcinoma cells. Cancer Lett. 2003;200:11521.

29. Gong K, Li W. Shikonin, a Chinese plant-derived naphthoquinone, induces apoptosis in hepatocellular carcinoma cells through reactive oxygen species: A potential new treatment for hepatocellular carcinoma. Free Radic Biol Med. 2011;51:2259-71.

30. Liu Y, Kang X, Niu G, He S, Zhang T, Bai Y, Li Y, Hao H, Chen C, Shou Z, Li B. Shikonin induces apoptosis and prosurvival autophagy in human melanoma A375 cells via ROS-mediated ER stress and p38 pathways. Artif Cells Nanomed Biotechnol. 2019;47:626-35.

31. Li D, Zhang T, Gu W, Li P, Cheng X, Tong T, Wang W. The ALDH1(+) subpopulation of the human NMFH-1 cell line exhibits cancer stem-like characteristics. Oncol Rep. 2015;33:2291-8.

32. Vargova J, Mikes J, Jendzelovsky R, Mikesova L, Kucharova B, Culka L, Fedr R, Remsik J, Soucek K, Kozubik A, Fedorocko P. Hypericin affects cancer side populations via competitive inhibition of BCRP. Biomed Pharmacother. 2018;99:511-22.

33. Januchowski R, Wojtowicz K, Sterzyska K, Sosiska P, Andrzejewska M, Zawierucha P, Nowicki M, Zabel M. Inhibition of ALDH1A1 activity decreases expression of drug transporters and reduces chemotherapy resistance in ovarian cancer cell lines. Int J Biochem Cell Biol. 2016;78:248-59.

34. Loblaw DA, Virgo KS, Nam R, Somerfield MR, Ben-Josef E, Mendelson DS, Middleton R, Sharp SA, Smith TJ, Talcott J, et al. Initial hormonal management of androgen-sensitive metastatic, recurrent, or progressive prostate cancer: 2006 update of an American Society of Clinical Oncology practice guideline. J Clin Oncol. 2007;25:1596-605.

35. Hellerstedt BA, Pienta KJ. The current state of hormonal therapy for prostate cancer. CA Cancer J Clin. 2002;52:154-79.

36. Liu J, Qu CB, Xue YX, Li Z, Wang P, Liu YH. MiR-143 enhances the antitumor activity of shikonin by targeting BAG3 expression in human glioblastoma stem cells. Biochem Biophys Res Commun. 2015;468:105-12.

37. Kuo LJ, Huang CY, Cheng WL, Hung CS, Wu CT, Lin FY, Chang YJ, Huang MT. Glucose-regulated protein 78 mediates the anticancer efficacy of shikonin in hormone-refractory prostate cancer cells. Tumour Biol. 2015;36:5063-70.

38. Di C, Zhao Y. Multiple drug resistance due to resistance to stem cells and stem cell treatment progress in cancer (Review). Exp Ther Med. 2015;9:289-93.

39. Donnenberg VS, Donnenberg AD. Multiple drug resistance in cancer revisited: the cancer stem cell hypothesis. J Clin Pharmacol. 2005;45:872-7.

40. Gara RK, Srivastava VK, Duggal S, Bagga JK, Bhatt M, Sanyal S, Mishra DP. Shikonin selectively induces apoptosis in human prostate cancer cells through the endoplasmic reticulum stress and mitochondrial apoptotic pathway. J Biomed Sci. 2015;22:26.

41. Rabindran SK, Ross DD, Doyle LA, Yang W, Greenberger LM. Fumitremorgin C reverses multidrug resistance in cells transfected with the breast cancer resistance protein. Cancer Res. 2000;60:47-50. 
42. Ricci JW, Lovato DM, Severns V, Sklar LA, Larson RS. Novel ABCG2 Antagonists Reverse TopotecanMediated Chemotherapeutic Resistance in Ovarian Carcinoma Xenografts. Mol Cancer Ther. 2016;15:2853-62.

43. Chen Y, Koppaka V, Thompson DC, Vasiliou V. Focus on molecules: ALDH1 A1: from lens and corneal crystallin to stem cell marker. Exp Eye Res. 2012;102:105-6.

44. Yan J, De Melo J, Cutz JC, Aziz T, Tang D. Aldehyde dehydrogenase 3A1 associates with prostate tumorigenesis. Br J Cancer. 2014;110:2593-603.

45. Parajuli B, Fishel ML, Hurley TD. Selective ALDH3A1 inhibition by benzimidazole analogues increase mafosfamide sensitivity in cancer cells. J Med Chem. 2014;57:449-61.

46. Okazaki S, Shintani S, Hirata Y, Suina K, Semba T, Yamasaki J, Umene K, Ishikawa M, Saya H, Nagano 0 . Synthetic lethality of the ALDH3A1 inhibitor dyclonine and $\mathrm{XCT}$ inhibitors in glutathione deficiency-resistant cancer cells. Oncotarget. 2018;9:33832-43.

47. Jia L, Zhu Z, Li H, Li Y. Shikonin inhibits proliferation, migration, invasion and promotes apoptosis in $\mathrm{NCl}$-N87 cells via inhibition of PI3K/AKT signal pathway. Artif Cells Nanomed Biotechnol. 2019;47:2662-9.

48. Li B, Yuan Z, Jiang J, Rao Y. Anti-tumor activity of Shikonin against afatinib resistant non-small cell lung cancer via negative regulation of PI3K/Akt signaling pathway. Biosci Rep 2018, 38.

49. Wang L, Lin N, Li Y. The PI3K/AKT signaling pathway regulates ABCG2 expression and confers resistance to chemotherapy in human multiple myeloma. Oncol Rep. 2019;41:1678-90.

50. Hu CF, Huang YY, Wang YJ, Gao FG. Upregulation of ABCG2 via the PI3K-Akt pathway contributes to acidic microenvironment-induced cisplatin resistance in A549 and LTEP-a-2 lung cancer cells. Oncol Rep. 2016;36:455-61.

\section{Figures}


a

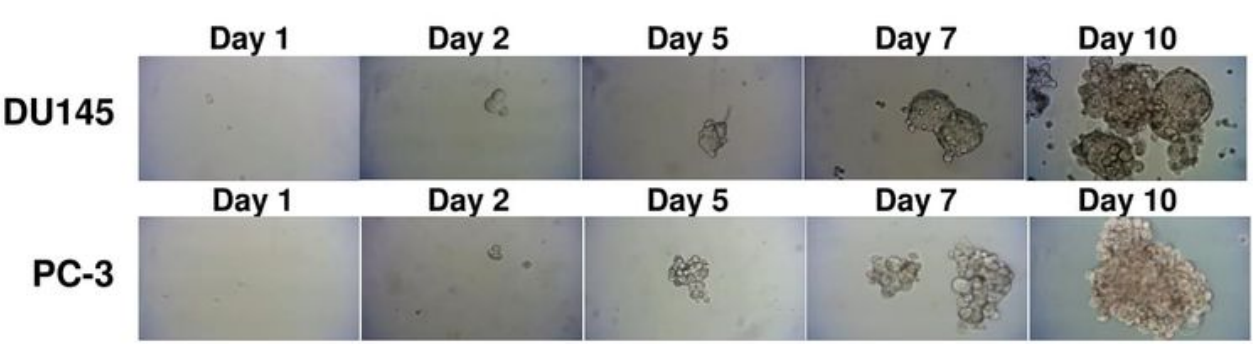

b
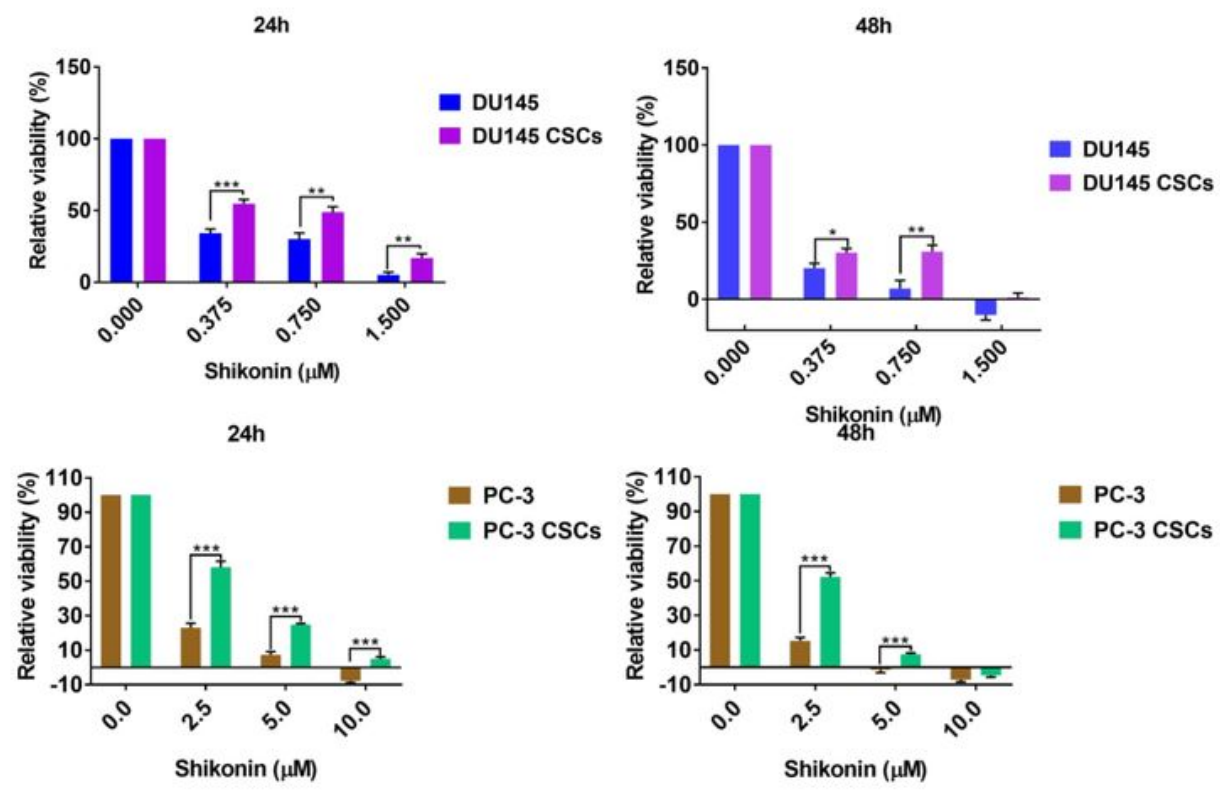

c
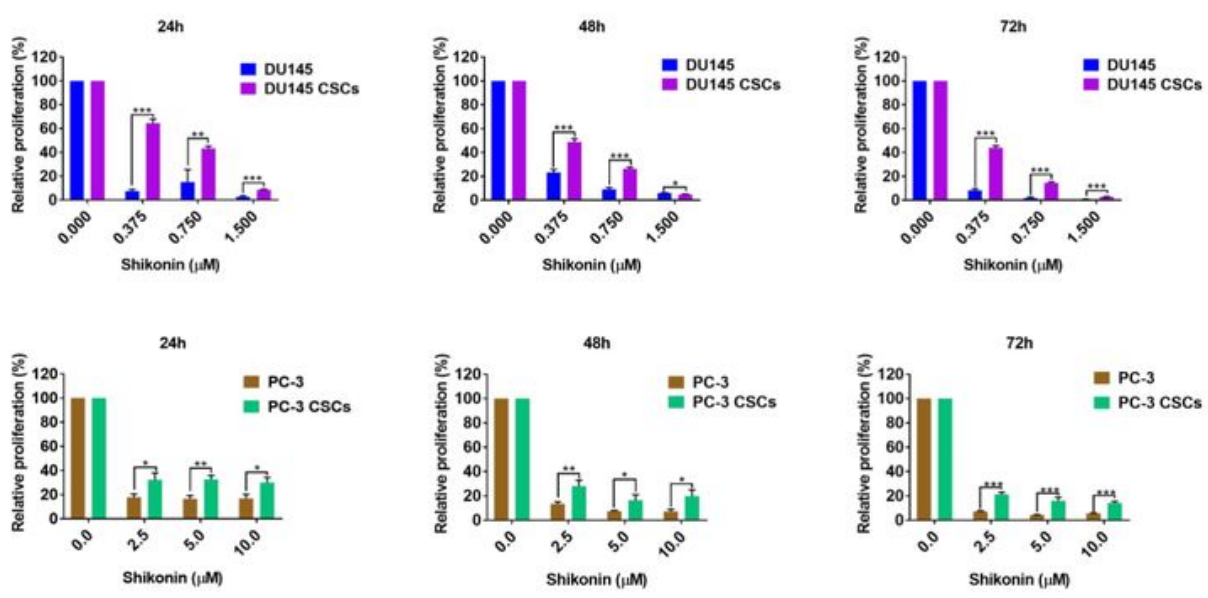

Fig. 1

\section{Figure 1}

Shikonin inhibits the viability and proliferation of PCa cells and PCSCs. a Sphere formation assay: DU145 and PC-3 cells formed spheres around day 7-10. Photos were taken by a microscope digital camera at the magnification of 100x (Bresser GmbH DE-46414 Rhede Germany). b CellTiter-Blue Cell Viability Assay: shikonin inhibited the viability of DU145, PC-3, and their corresponding PCSCs in a dose-dependent manner, shown for 24 and 48 hours. c CellTiter 96 AQueous One Solution Cell Proliferation Assay: 
shikonin inhibited the proliferation of DU145, PC-3, and their corresponding PCSCs, shown for 24,48 , and 72 hours. The results are presented as the means \pm SEM of values obtained in three independent experiments. ${ }^{*} p<0.05 ;{ }^{* *} p<0.01 ;{ }^{* *} p<0.001$.
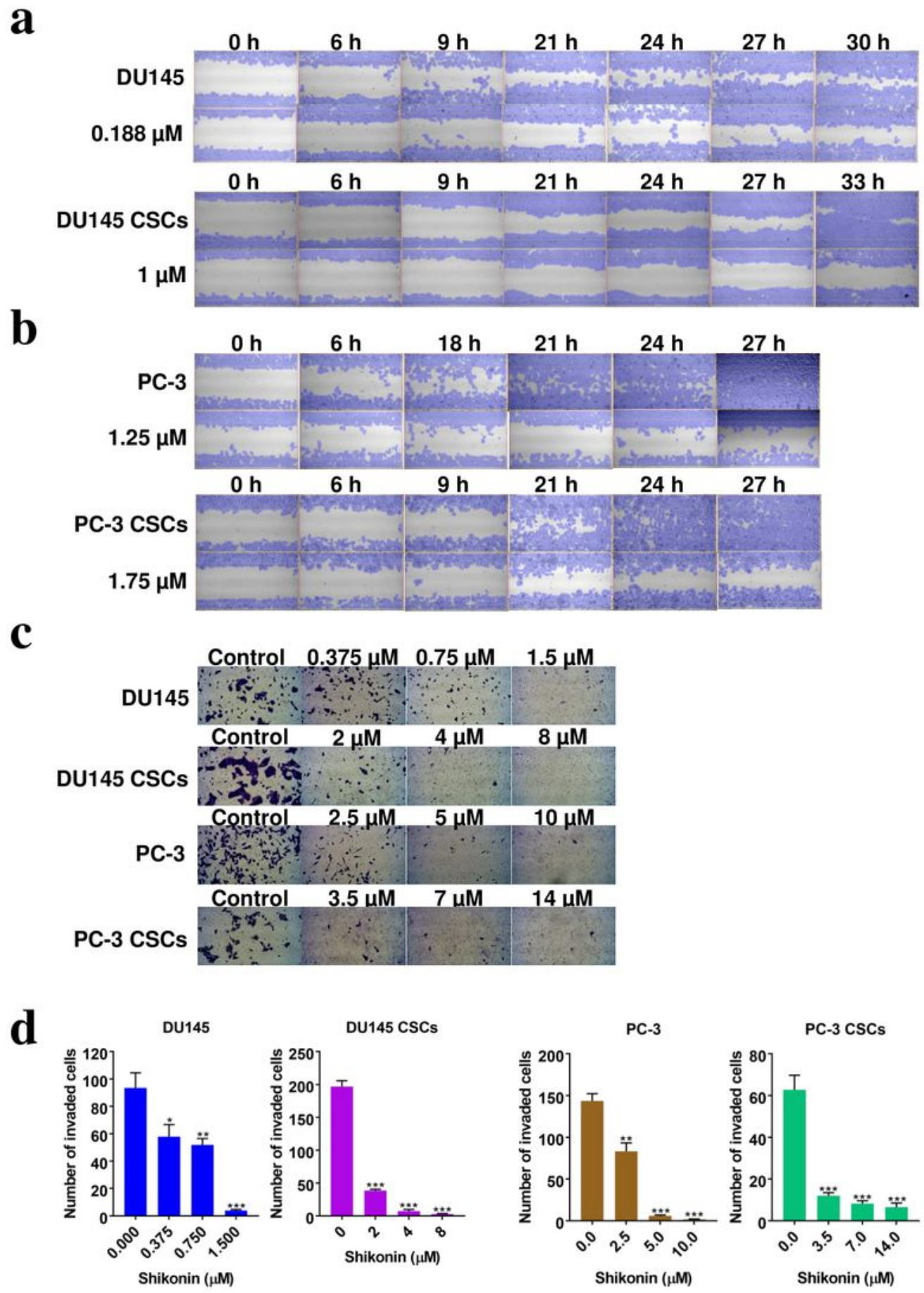

Fig. 2

Figure 2

Shikonin inhibits migration and invasion in PCa cells and PCSCs. a and b Scratch wound healing assay. Plates with a cell free gap of $500 \square \mathrm{m}$ were used (ibidi $\mathrm{GmbH}$ ): shikonin inhibited the migration ability of 
DU145, DU145 CSCs, PC-3, PC-3 CSCs. Photos were taken under the microscope at different time points at the magnification of $40 \times$ (Bresser GmbH DE-46414 Rhede Germany). The data were analyzed with the web-based Automated Cellular Analysis System (ACAS, MetaVi Labs) using FastTrack Al image analysis algorithms. c Invasion assay using the Boyden Chamber system with transwell inserts in 24-well plates (8.0 $\mu \mathrm{m}$ pores), coated with growth factor reduced Matrigel Basement Matrix: shikonin inhibited the invasive ability of DU145, DU145 CSCs, PC-3, and PC-3 CSCs through the Matrigel. From every insert a photo was taken by the camera at the magnification of $40 \times$ and the cells were counted (three fields per insert; Fiji ImageJ software). d The number of invaded cells for DU145, DU145 CSCs, PC-3, and PC-3 CSCs were shown. The results are presented as the means \pm SEM of values obtained in three independent experiments. ${ }^{\star} \mathrm{p}<0.05 ;{ }^{* \star} \mathrm{p}<0.01 ;{ }^{* * *} \mathrm{p}<0.001$. 

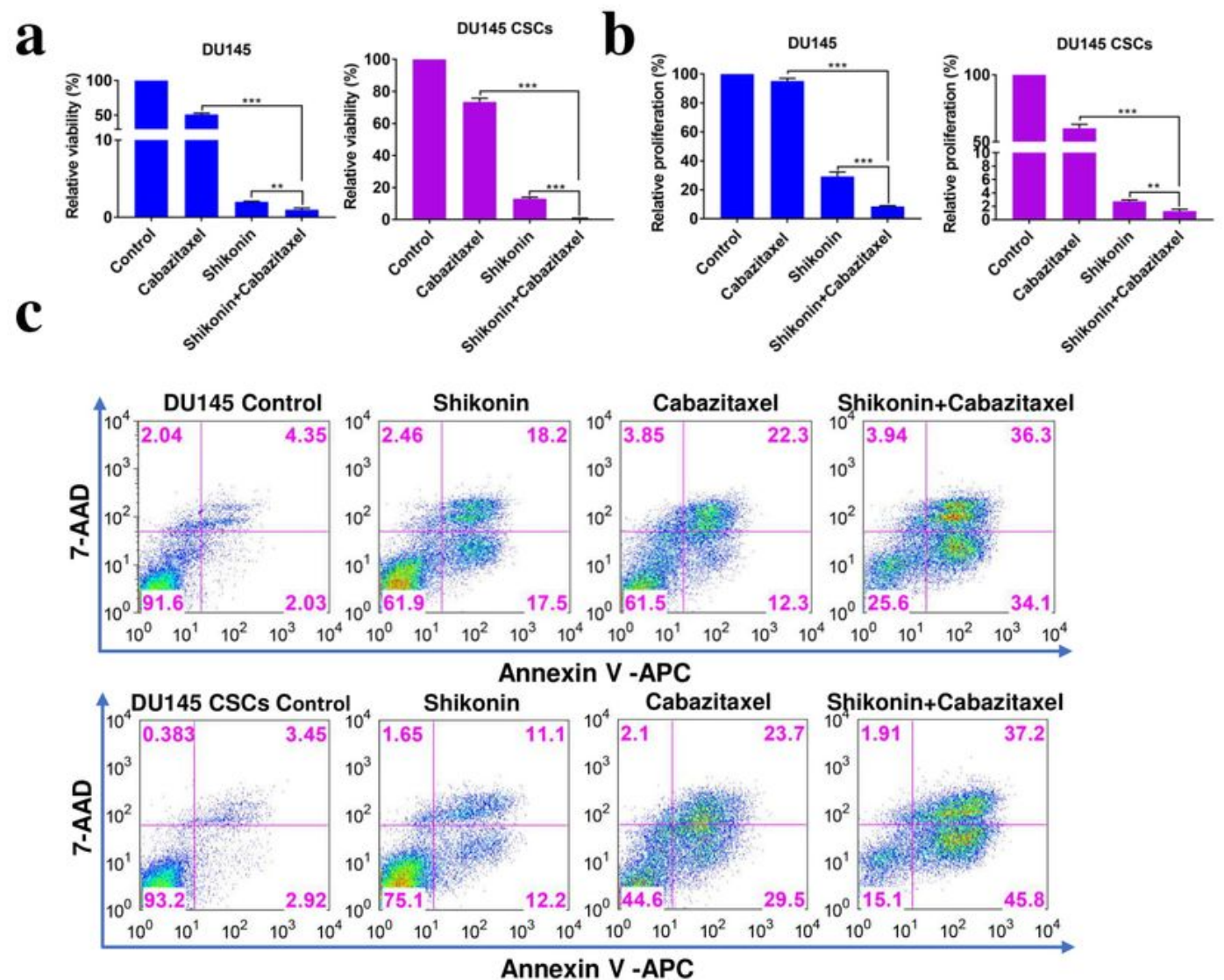

d

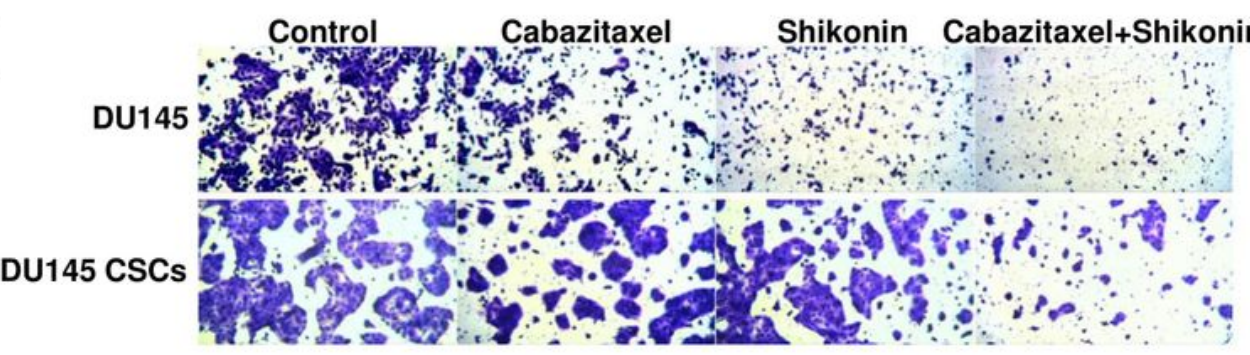

DU145

DU145 CSCs

Fig. 3

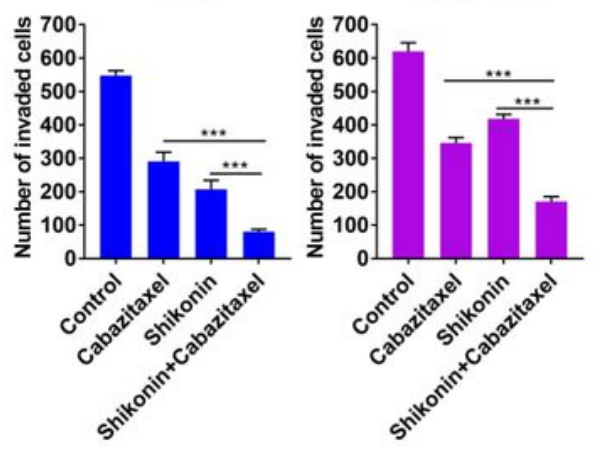

\section{Figure 3}

Shikonin enhances the antitumor effect of cabazitaxel in DU145 cells and DU145 CSCs. a CellTiter-Blue Cell Viability Assay: combination of shikonin $(0.75 \mathrm{\square M})$ and cabazitaxel $(0.75 \mathrm{nM})$ enhanced the antitumor effect compared with cabazitaxel or shikonin alone. The viability was measured after 48 hours. b CellTiter 96 AQueous One Solution Cell Proliferation Assay: combination of shikonin (0.75 $\mathrm{MM})$ and cabazitaxel $(0.75 \mathrm{nM})$ decreased the proliferation ability compared with cabazitaxel or shikonin alone. 
The proliferation was determined after 48 hours. c Apoptosis was measured by Annexin IV and 7-AAD staining and flow cytometry: shikonin in combination with cabazitaxel enhanced the apoptosis rate in DU145 cells and the corresponding DU145 CSC d Invasion assay: combination of shikonin and cabazitaxel decreased the invasion ability of DU145 cells and DU145 CSCs compared with cabazitaxel or shikonin alone. The results are presented as the means \pm SEM of values obtained in three independent experiments. ${ }^{\star} p<0.05 ;{ }^{* \star} p<0.01 ;{ }^{* \star *} \mathrm{p}<0.001$.
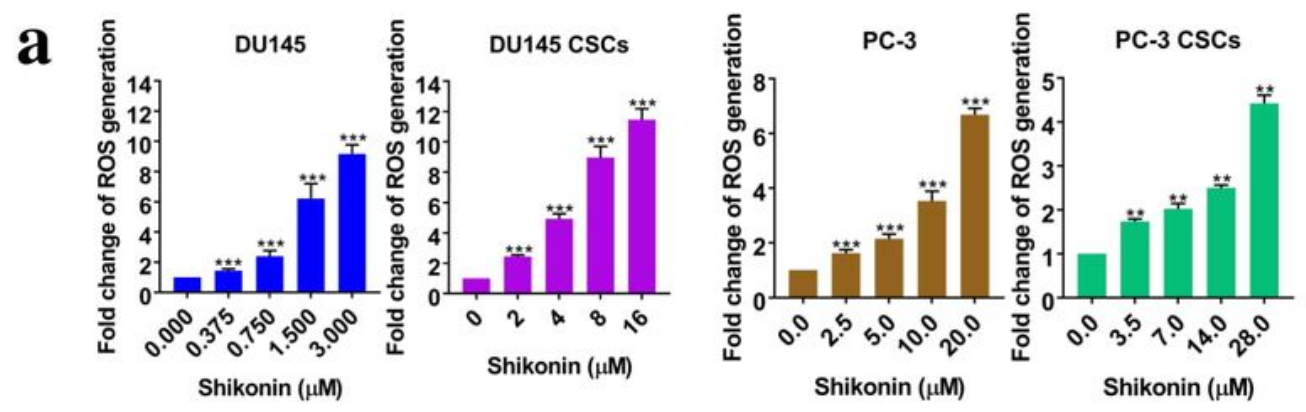

b
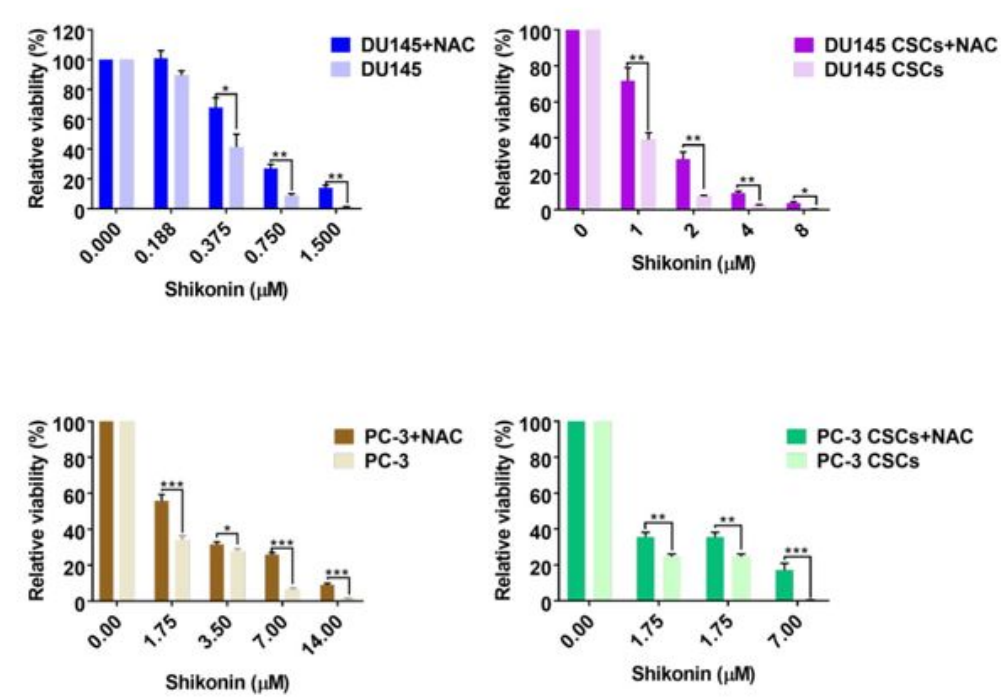

c
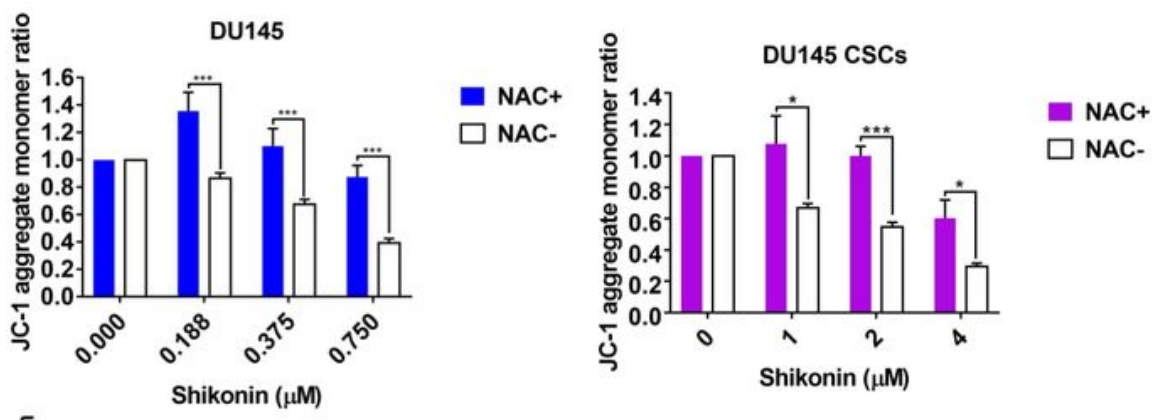

Fig. 4

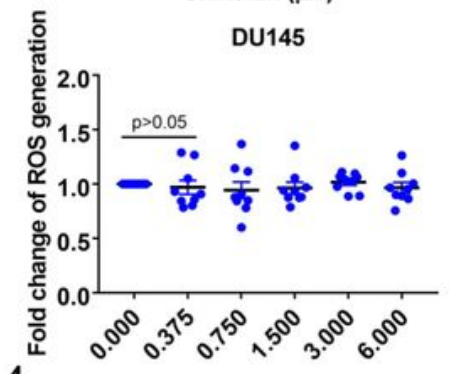

Figure 4 
Shikonin induces ROS dependent mitochondrial apoptosis in the PCa cell lines and the corresponding PCSCs. a Shikonin induced ROS generation in DU145, DU145-CSC, PC-3, and PC-3 CSC cells in a concentration dependent manner. $b$ The viability assay showed that ROS generation was rescued by the antioxidant $\mathrm{N}$-acetyl-L-cysteine (NAC) after pretreatment for 4 hours at $1 \mathrm{mM}$. c The integrity of mitochondrial membrane potential $(\Delta \psi \mathrm{m})$ was evaluated using the JC-1 mitochondrial membrane potential kit. JC-1 dye staining indicated that shikonin dysregulated the mitochondrial membrane potential along with higher concentrations. NAC pretreatment caused the inhibition of mitochondria membrane potential dysregulation. $d$ Cabazitaxel cannot influence ROS generation. The results are presented as the means \pm SEM of values obtained in three independent experiments. ${ }^{*} p<0.05 ;{ }^{* \star} p<0.01$; $\star \star \star p<0.001$. 


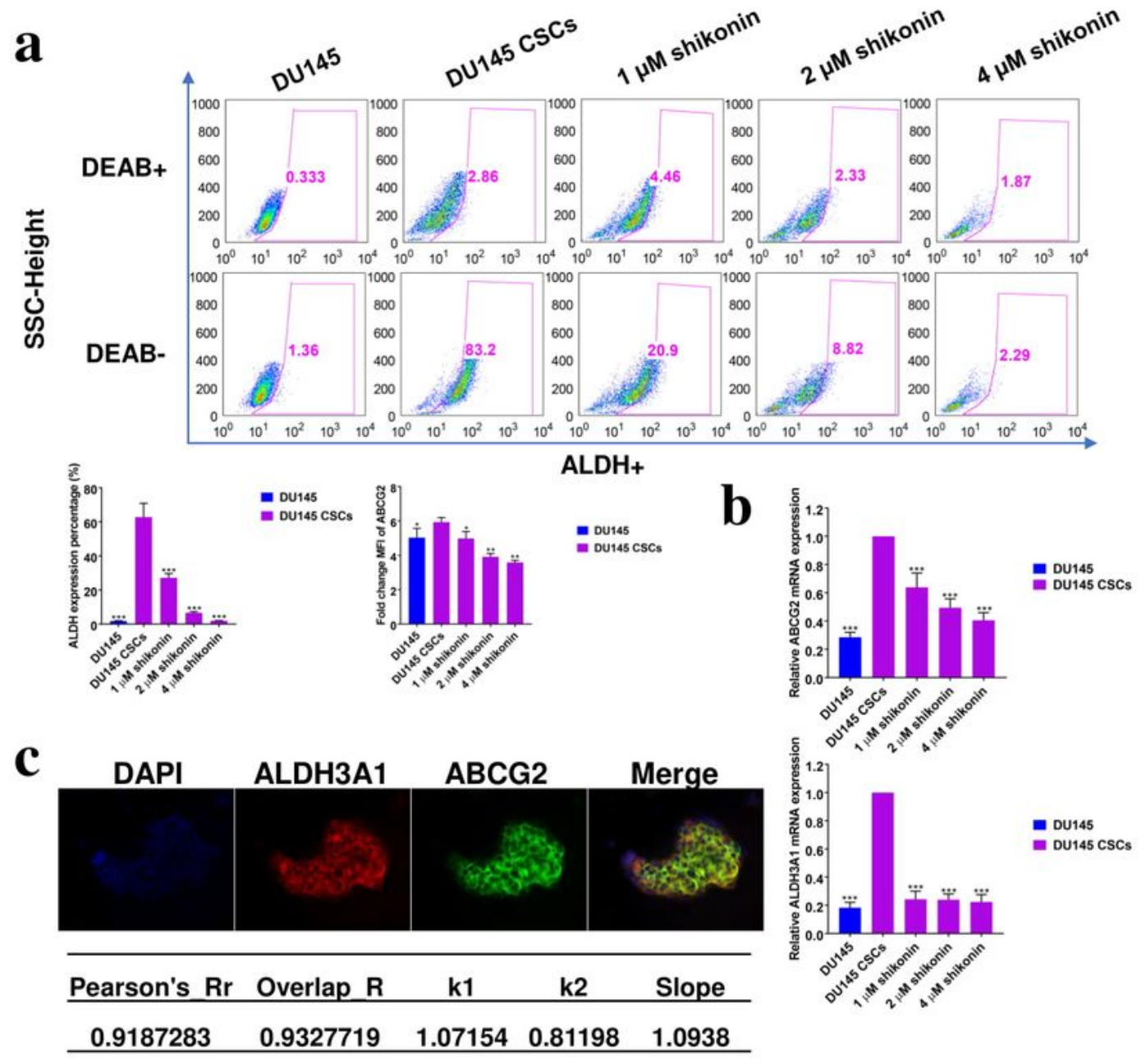

d
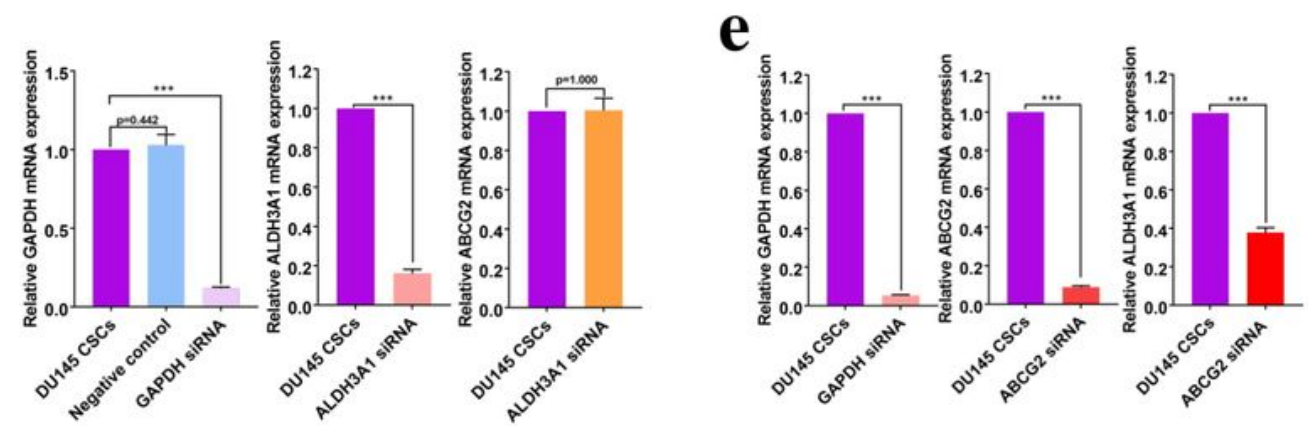

Fig. 5

\section{Figure 5}

Shikonin inhibits the expression of ABCG2 and ALDH3A1 in PCSCs. a ALDEFLUOR kit was used to measure the expression of ALDH in DU145 and DU145 CSCs. As suspected DU145 CSCs expressed more ALDH than DU145 cells. The ALDH inhibitor N,N-diethylaminobenzaldehyde (DEAB)was used as control. b Flow cytometry: shikonin inhibited the expression of ABCG2 along with higher concentrations. $C$ Confocal immunofluorescence microscopy showed co-expression of ALDH3A1 and ABCG2 on DU145 
CSCs. Both Pearson's correlation and overlap coefficients between ALDH3A1 and ABCG2 were 0.9. d RTqPCR analysis: silencing ALDH3A1 by specific siRNA cannot interrupt the expression of ABCG2. GAPDH siRNA was used as positive control. e Silencing ABCG2 by specific siRNA decreased the expression of ALDH3A1. GAPDH siRNA was used as positive control. The results are presented as the means \pm SEM of values obtained in three independent experiments. ${ }^{*} p<0.05 ; * * p<0.01 ; * * p<0.001$.

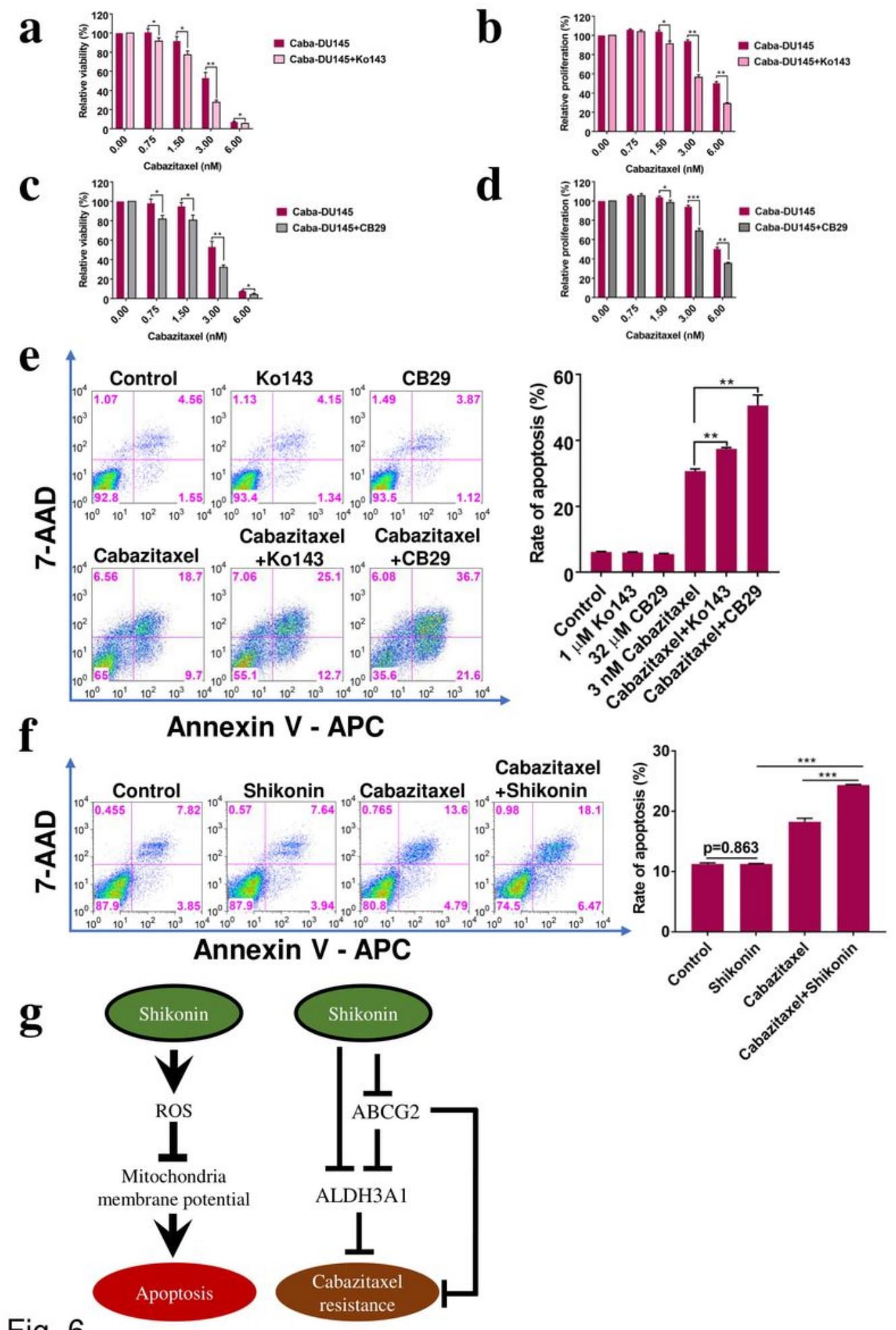

Fig. 6

Figure 6 
Shikonin reverses the resistant-state of caba-DU145 through inhibiting the expression of ABCG2 and ALDH3A1. $a$ and b Pretreatment of caba-DU145 cells with ABCG2 inhibitor Ko143 for 7 days led to an increased cabazitaxel sensitivity as shown by the viability assay and the proliferation assay. $c$ and $d$ Pretreatment of caba-DU145 cells with ALDH3A1 inhibitor CB29 for 7 days led also to an increased sensitivity against cabazitaxel. The caba-DU145 cells were treated with cabazitaxel for another 72 hours after pretreatment with Ko143 and CB29. e Annexin V and 7-AAD staining demonstrated an enhanced rate of apoptosis in caba-DU145 cells following treatment with Ko143 and CB29 compared to cabazitaxel ( $3 \mathrm{nM}$ ) alone. Ko143 and CB29 alone cannot induce apoptosis. $\mathrm{f}$ Combination of shikonin with cabazitaxel increased the rate of apoptosis in caba-DU145 cells based on the annexin V and 7-AAD staining. $g$ Proposed model for apoptosis induction by shikonin and the mechanism of reversing the cabazitaxel-related resistance. The results are presented as the means \pm SEM of values obtained in three independent experiments. ${ }^{*} p<0.05 ;{ }^{* \star} p<0.01 ; * \star * p<0.001$.

\section{Supplementary Files}

This is a list of supplementary files associated with this preprint. Click to download.

- Additionalfile10FigureS8Wangetal15.07.2020.pdf

- Additionalfile9FigureS7Wangetal15.07.2020.pdf

- Additionalfile8FigureS6Wanget115.07.2020.pdf

- Additionalfile7Figures5Wangetal15.07.2020.pdf

- Additionalfile6FigureS4Wangetal15.07.2020.pdf

- Additionalfile5FigureS3Wangetal15.07.2020.pdf

- Additionalfile4FigureS2Wangetal15.07.2020.pdf

- Additionalfile3FigureS1Wangetal15.07.2020.pdf

- Additionalfile2TableS2Wangetal15.07.2020.pdf

- Additionalfile1TableS1Wangetal15.07.2020.pdf 